\title{
The nonlinear downstream development of baroclinic instability
}

\author{
by Joseph Pedlosky ${ }^{1}$
}

\begin{abstract}
The downstream development in both space and time of baroclinic instability is studied in a nonlinear channel model on the $f$-plane. The model allows the development of the instability to be expressed on space and time scales that are long compared to the growth rates and wavelengths of the most unstable wave. The unstable system is forced by time-varying boundary conditions at the origin of the channel and so serves as a conceptual model for the development of fluctuations in currents like the Gulf Stream and Kuroshio downstream of their separation points from their respective western boundaries.

The theory is developed for both substantially dissipative systems as well as weakly dissipative systems for which the viscous decay time is of the order of the advective time in the former case and the growth time in the latter case. In the first case a first order equation in time leads to a hyperbolic system for which exact solutions are found in the case of monochromatic forcing. For a finite bandwidth the governing equations are nonlinear and parabolic and could be put in the form of the Real Ginzburg Landau equation first developed by Newell and Whitehead (1969) and Segel (1969) although we show the equation is not pertinent to the downstream development problem.

When the dissipation is small a third order system of partial differential equations is obtained. For steady states the system supports chaotic behavior along the characteristics. This produces for the-time dependent problem new features, principally a strong focusing of amplitude in the regions behind the advancing front and the appearance of what might be called "chaotic shocks."
\end{abstract}

\section{Introduction}

The problem of the downstream development of linear and nonlinear instabilities has a long history in fluid mechanics. The subject was first developed in the context of the classical shear flow instability problem. Both linear and weakly nonlinear theoretical approaches to the problem were developed in that context (e.g. Stewartson and Stuart, 1971 and Hocking et al., 1972). In the area of oceanographic and geophysical fluid dynamic interest, studies such as Pedlosky (1976) and Polvani and Pedlosky(1988) have considered both the linear and weakly nonlinear aspects of the problem of the spatial development of perturbations in a baroclinic current. The subject has been developed considerably since those studies and a recent paper by Waterman and Jayne (2011) examines the spatial structure of growing

1. Woods Hole Oceanographic Institution, Woods Hole, Massachusetts, 02543, U.S.A. email: jpedlosky@whoi.edu 
instabilities and their interaction with the mean flow in a realistic oceanic setting involving the Kuroshio extension and its meandering path in the Pacific. The reader is also directed there for further references to the expanding literature on this subject. An excellent general review of baroclinic instability and the role of spatial instability and its connection to the question of local and global instabilities can be found in Pierrehumbert and Swanson (1995). The pioneering paper in this subject dates back to Briggs (1964) where the connection between the phenomenon of temporal growth and that of spatial growth was first discussed fully.

The focus of the present paper is on the development of our understanding through simple models with a sharp focus on the role of time-dependent upstream boundary conditions on the further evolution, both spatial and temporal, of growing disturbances. In an oceanographic context, such a model can be considered an idealized representation of the response of an unstable zonal extension of a western boundary current to naturally produced fluctuations at the separation latitude of the boundary current.

We consider two simple models, both without the presence of the beta-effect. The model is a two-layer model with a baroclinic current with no horizontal shear as in the early models of Pedlosky (1976) and Pedlosky and Polvani (1988). In contrast to those models the domain of the flow is semi-infinite $0 \leq x \leq \infty$, where $x$ is the downstream coordinate. Time-dependent boundary conditions are applied at the origin $x=0$ and the propagation of the resulting disturbance through the region of the unstable current is the focus of the discussion. The two models are considered different only in the degree of the dissipation in the system. The first model is sufficiently dissipative so that the marginal curve for instability is determined by friction while the second model, otherwise identical, is nearly inviscid.

Section 2 describes the formulation of the model. Section 3 treats the linear and nonlinear analysis of the frictional problem and describes the solution to the nonlinear problem in closed form along with a representation of the response. In particular a discussion of the large space scale response of the interface and vertical velocity are given. Section 4 takes up the problem of the nearly inviscid problem. The resulting equations in characteristic coordinates are presented and are one to one with the well-known Lorenz equations (Lorenz, 1963), and so the solutions along the characteristic curves can be chaotic. The problem of connecting the solutions far from the origin to the forcing at the origin is described and the behavior of the solution is discussed. The chaotic behavior along characteristics with its sensitivity to initial data implies that neighboring characteristics with similar but slightly different initial conditions can lead to regions of rapid variation in space at times when the solutions trajectories along neighboring characteristics strongly diverge. Section 5 concludes our study with a summary and discussion of the results.

\section{The model}

We consider the standard, two-layer, quasi-geostrophic model (Pedlosky, 1987). The layer thicknesses are taken to be equal in their rest state. In non-dimensional units the equations for the barotropic and baroclinic perturbation streamfunctions, $\varphi_{B}, \varphi_{T}$, respectively are 


$$
\begin{aligned}
& \left(\frac{\partial}{\partial t}+U_{B} \frac{\partial}{\partial x}\right) \nabla^{2} \varphi_{B}+\frac{U_{s}}{4} \frac{\partial}{\partial x} \nabla^{2} \varphi_{T}+J\left(\varphi_{B}, \nabla^{2} \varphi_{B}\right)+\frac{1}{4} J\left(\varphi_{T}, \nabla^{2} \varphi_{T}\right)=-r \nabla^{2} \varphi_{B} \\
& \left(\frac{\partial}{\partial t}+U_{B} \frac{\partial}{\partial x}\right)\left[\nabla^{2} \varphi_{T}-2 F \varphi_{T}\right]+U_{s} \frac{\partial}{\partial x}\left[\nabla^{2} \varphi_{B}+2 F \varphi_{B}\right]+J\left(\varphi_{B}, \nabla^{2} \varphi_{T}-2 F \varphi_{T}\right) \\
& +J\left(\varphi_{T}, \nabla^{2} \varphi_{B}\right)=-r \nabla^{2} \varphi_{T}
\end{aligned}
$$

where the barotropic and baroclinic streamfunctions are defined in terms of their layer equivalents $\varphi_{B}=\frac{1}{2}\left(\varphi_{1}+\varphi_{2}\right), \varphi_{T}=\varphi_{1}-\varphi_{2}$. The parameter $F=\frac{f^{2} L^{2}}{g^{\prime} H}$ where $L$ is the width of the channel in which the flow is contained, and $H$ is the rest thickness of each layer. The parameter $r$ is a measure of the spin-down time due to Ekman layers with respect to the advection time of the flow. The coordinate down the channel in the direction of flow is $x$ while $y$ is the cross channel coordinate, $0 \leq y \leq 1$. The barotropic part of the basic flow is $U_{B}$ while the shear of the basic flow is $U_{s}=U_{1}-U_{2}$. It is important to recall that the departure from the rest state of the interface is proportional to $-\varphi_{T}$.

Standard linear stability analysis yields a marginal condition for the shear that must be exceeded for instability to occur. For example, for $r=O(1)$ the condition for instability is that the shear of the basic flow must exceed the value,

$$
U_{s}=\frac{2 r K / k}{\left(2 F-K^{2}\right)^{1 / 2}}
$$

where $k$ is the $x$ wavenumber and $K$ is the total wavenumber, $K=\left(k^{2}+l^{2}\right)^{1 / 2}$ and where $l$, the cross-stream wavenumber, is quantized in integer multiples of $\pi$. We will consider shears that are greater than the critical shear by an amount $\Delta$, examine the dynamics of disturbances in the channel forced by inhomogeneous conditions on the streamfunction at the origin, $x=0$, and examine the behavior of the resulting perturbation as function of time and position downstream of the origin. The boundary conditions in $y$ for $(2.1 \mathrm{a}, \mathrm{b})$ are that the velocity in the $y$ direction vanish at $y=0,1$ or that

$$
\frac{\partial}{\partial x}\left\{\begin{array}{c}
\varphi_{B} \\
\varphi_{T}
\end{array}\right\}=0, \quad y=0,1
$$

\section{The theory for $r=\mathrm{O}(1)$}

When $r$ is order one, the minimum critical shear, i.e. the minimum of $U_{S}$ as a function of $k$, occurs at $K^{2}=l(2 F)^{1 / 2}$.

The corresponding minimum critical shear is $U_{s o}=\frac{r}{(2 F)^{1 / 2}-l}, k=\left(K^{2}-l^{2}\right)^{1 / 2}$ at which $k$, the real frequency of the disturbance is simply $\omega=k U_{B}$. The theory will be developed in the neighborhood of this parameter setting but for the time being, one can think of the forcing at $x=0$ being at a single frequency $\omega$ corresponding to the most unstable wave. In the standard fashion we can introduce "slow" time and space coordinates

$$
T=|\Delta| t, X=|\Delta x|, U_{s}=U_{s o}+\Delta .
$$


We also introduce a scaling for the geostrophic streamfunctions. We choose the nondimensional amplitude of the streamfunction to be $\varepsilon$ and consider the expansion of both the barotropic and baroclinic perturbations in an asymptotic series in $\varepsilon$, i.e.

$$
\varphi=\varepsilon\left[\varphi^{(0)}+\varepsilon \varphi^{(1)}+\varepsilon^{2} \varphi^{(2)}+\ldots\right]
$$

and where each function is a function of $x, t, X, T$. We shall note later that intermediate time and space scales are possible but will for the time being consider disturbances with just these scales. In order to carry out a well ordered expansion, a relation between $\Delta$ and $\varepsilon$ is required. As in earlier studies, the proper relation is $\varepsilon=O\left(\Delta^{1 / 2}\right)$ and that relation allows a straightforward expansion of $(2.1 \mathrm{a}, \mathrm{b})$ in powers of $\varepsilon$ in which the ratio $\Delta^{1 / 2} / \varepsilon$ will enter as a parameter as an (inverse) measure of nonlinearity.

At lowest order the solution can be written,

$$
\begin{aligned}
\varphi_{B} & =\frac{1}{2} A_{B} e^{i(k x-\omega t)} \sin l y+*, \\
\varphi_{T} & =\frac{1}{2} A_{T} e^{i(k x-\omega t)} \sin l y+*,
\end{aligned}
$$

where $k$ and $\omega$ are the wavenumber and frequency of the marginal stable solution at the minimum critical shear. The asterisks in (3.3) denote the complex conjugate of the preceding term. The solution at the marginal curve determines a relation between the amplitudes of the barotropic and baroclinic streamfunction, namely,

$$
A_{T}=i \frac{4 r}{k U_{s o}} A_{B} .
$$

It is important to note that the amplitudes are both functions, as yet unknown, of both $T$ and $X$.

At the next order the self interaction of the lowest order solution forces a correction to the basic flow. The correction satisfies,

$$
\frac{\partial^{2}}{\partial y^{2}} \varphi_{T}^{(1)}=\frac{4 K^{2} l}{U_{s o}} F \sin 2 l y\left|A_{B}\right|^{2} .
$$

Two interesting features of (3.5) are that although the forcing is due to the phase shift in the marginal wave between the barotropic and baroclinic parts of the wave, and that the phase shift is due to the presence of friction, (3.4), the correction to the mean flow is independent of the friction except as it manifests itself in the behavior of the barotropic amplitude. Second, the forcing of the mean flow correction depends on the cross-stream mode number. For an infinite region the most unstable mode would have $l=0$ and there would be in that singular case no nonlinear mean flow correction.

The solution to (3.5) is

$$
\varphi_{T}^{(1)} \equiv \Phi_{T}=-\frac{F}{l U_{s o}} \sin 2 l y\left|A_{B}\right|^{2}+C_{T} y+D_{T}
$$


where all the "constants" in (3.6) are, in fact, functions of $X$. The derivative of the mean flow correction depends only on the variation in the slow space variable $X$ and hence is order $\Delta$. The ageostrophic velocity proportional to the acceleration of this geostrophic mean flow correction and is of order $R_{o} \Delta$ where $R_{o}$ is the Rossby number. At the same time, the frictional contribution to the zonal momentum equation and, hence, the ageostrophic meridional velocity, is order $R_{O} r$ and it is the dominant ageostrophic term. Hence as long as the ordering relationship $1 \gg \Delta \gg R_{o}$ holds, to have no meridional velocity at the channel boundaries, the solution (3.6) must satisfy the condition that

$$
\frac{\partial}{\partial X} \Phi_{T}=0, \quad y=0,1
$$

and so both $C_{\mathrm{T}}$ and $D_{\mathrm{T}}$ must vanish in distinction to the case of a mean flow correction in an infinitely long channel with a purely zonal mean flow correction. Thus

$$
\Phi_{T}=-\frac{F}{l U_{s}} \sin 2 l y\left|A_{B}\right|^{2}
$$

and note that the solution has zero average across the channel for all $X$. Since $-\Phi_{T}$ is proportional to the large scale deformation of the interface due to the instability, the overall rise or fall of the interface must be zero at each value of $X$.

At the next order, i.e. $O(\Delta)=O\left(\varepsilon^{2}\right)$ the expansion first recognizes the excess shear above the critical value as well as the variations of the lowest order streamfunctions on the slow space and time scales as well as the interaction of the lowest order solutions with the correction to the mean flow. Some of these terms would force a resonant response in the order $\varepsilon^{2}$ solutions. The condition to eliminate these resonant terms yields as a condition to maintain the validity of our original expansion a equation for the behavior of $A_{B}$ and hence $A_{T}$ on the slow space and time scales, namely,

$$
\begin{aligned}
& \left(\frac{\partial}{\partial T}+U_{B} \frac{\partial}{\partial X}\right) A_{B}-\sigma A_{B}+N A\left|A_{B}\right|^{2}=0, \\
& \sigma=\frac{\Delta}{|\Delta|} \frac{k K}{2} \frac{\left(2 F-K^{2}\right)^{1 / 2}}{F+K^{2}} \\
& N=\frac{\varepsilon^{2}}{|\Delta|} \frac{F k}{8 r}\left(2 K^{2}\left\{2 F-K^{2}\right\}-8 l^{2}\left(F-K^{2}\right)\right) /\left(2 F-K^{2}\right)
\end{aligned}
$$

It is easy to show that at the minimum critical shear, where this analysis is valid, the nonlinear Landau coefficient, $N$, is positive.

Eq. (3.9a) can be easily solved using the method of characteristics. The characteristic curves, shown in Figure 1 are the straight lines $X-U_{B} T=$ const. and the line for which $X=U_{B} T$ separates those characteristics that intersect the $X$ axis from those that intersect the $T$ axis. It also represents the wave front carrying information of the forcing at $X=0$. 


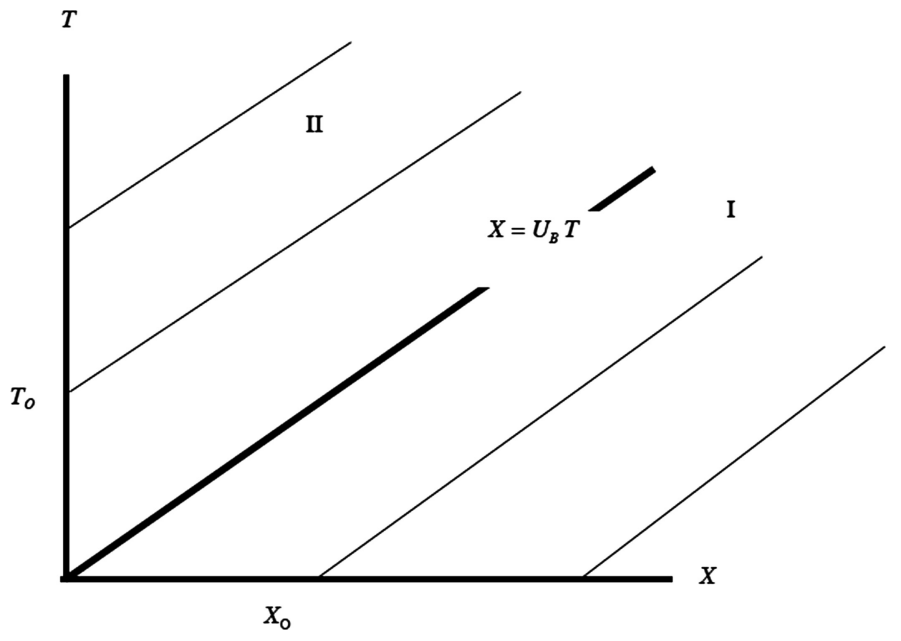

Figure 1. The characteristics in the $X, T$ plane. Each characteristic has a slope $U_{B}^{-1}$. The characteristics in region II intersect the $T$ axis at points labeled $T_{o}$ while they intersect the $X$ axis at points $X_{O}$ in region I. Boundary and initial data are given at those points.

The solution can be found in closed form. For $X<U_{B} T$, i.e. behind the advancing front (region II in Fig. 1), the solution for $Z=A_{B}^{2}$ can be written

$$
\begin{aligned}
& X<U_{B} T \\
& Z=\frac{Z_{o}\left(T_{o}\right) e^{2 \sigma X / U_{B}}}{1+\frac{N}{\sigma} Z_{o}\left(T_{o}\right)\left(e^{2 \sigma X / U_{B}}-1\right)}=\frac{Z_{o}\left(T-X / U_{B}\right) e^{2 \sigma X / U_{B}}}{1+\frac{N}{\sigma} Z_{o}\left(T-X / U_{B}\right)\left(e^{2 \sigma X / U_{B}}-1\right)}
\end{aligned}
$$

where $Z_{o}\left(T_{o}\right)$ is the square of the barotropic wave amplitude at the origin $X=0$, as a function of the time $T_{o}=T-X / U_{B}$. The solution ahead of the front $X>U_{B} T$ (region I in Figure 1) is determined by the initial data, $Z_{I}\left(X_{o}\right)$ given as a function of $X$ at $T=0$.

$$
\begin{aligned}
& X>U_{B} T \\
& Z=\frac{Z_{I}\left(X-U_{B} T\right) e^{2 \sigma T}}{1+Z_{I} \frac{N}{\sigma}\left(e^{2 \sigma T}-1\right)}
\end{aligned}
$$

Note that the solution will be continuous across the front as long as $Z_{o}(0)=Z_{I}(0)$. For large $T$ ahead of the front or for large $X / U_{B}$ behind the front there is a tendency for the solution to reach its equilibrium value $\sigma / N$ (assuming, as in our case that $N>0$ ). It also follows from the fact that the coefficients of (3.9a) are real, that the phase of $A_{B}$ remains constant along a characteristic and is completely determined by the starting conditions, for example, if we write $A_{B}=Z^{1 / 2} e^{i \theta}$, then in region II, $\theta=\theta_{o}\left(T-X / U_{B}\right)$. Henceforth we will consider solutions to (3.9) that are real without essential loss of generality. In fact 
by scaling $T$ with $\sigma$ and $X$ with $\sigma / U_{B}$ and the amplitude with the square root of $\sigma / N$ all parameters are eliminated from (3.9) and we can consider the coefficients in (3.9) to be unity. The only remaining parameters will be the parameters measuring the properties of the initial and boundary conditions.

Figure 2 shows the solution for the conditions (in our scaled units)

$$
\begin{aligned}
A_{B} & =Y_{o}\left(1+a_{o} \cos (\omega T)\right) /\left(1+a_{o}\right), & X & =0 . \\
& =Y_{o} e^{-2 q X}, & T & =0
\end{aligned}
$$

Thus, at the origin $(X=0, T=0)$ the amplitude is $Y_{o}$ and so the solution will be continuous across the characteristic emanating from the origin. Recall that this amplitude is with respect to the equilibrium amplitude $(\sigma / N)^{1 / 2}$ which is unity in our scaled units. It also oscillates at $X=0$ with a period $2 \pi / \omega$, whose time scale is measured in e-folding growth times of the linear problem. As an initial condition a disturbance exponentially decreasing from the origin is specified with a decay scale $q^{-1}$. The solution for $A_{B}$ is shown in Figure 2a. The front, i.e. the position $X=U_{B} T$ is, in the scaled units described above, at $X=4$. Ahead of the front the small initial disturbance grows rapidly, initially according to linear theory, such that when the front arrives the disturbance has reached finite amplitude and has already reached its equilibrium amplitude, unity in our scaled units. Behind the front the oscillations imposed at the origin are evident and the spatial growth of the amplitude as the disturbance moves downstream follows the result in (3.10) as it tends towards the equilibrium amplitude that is independent of the boundary data. The smaller the amplitude is at $X=0$ (it is 0.2 in the example), the larger the space occupied by the region behind the front reflecting the behavior at the origin. Thus, even though the amplitude equation is first order in time and space the oscillations imposed at the boundary remain a significant part of the spatial structure of the downstream development.

Figure $2 \mathrm{~b}$ shows the correction to the mean streamfunction given by (3.5) for the amplitude shown in panel (a). This baroclinic streamfunction is zero in the mid latitude of the channel so that the stream field represents two counter rotating circulations. At the same time it also, with a change in sign, represents the response on the slow time and space scales of the interface between the two layers. The response is antisymmetric about the mid line of the channel so that as the disturbance advances a depression of the interface at $y$ is balanced by an elevation at $1-y$. Hence there is no net change in the cross channel averaged depth and no net vertical motion. Although the growing baroclinic instability reduces the shear in the middle of the channel, and so increasing the flow in the lower layer there; the opposite occurs on the flanks of this region so there is no net increase or decrease in the zonal transport at any zonal position. Of course, this must be the case since we have allowed no cross interface transport in our model.

There is one further important consideration to discuss in this parameter regime. The critical curve of $U_{S}$ as a function of $k$ is parabolic at its minimum. Thus, for a supercriticality 

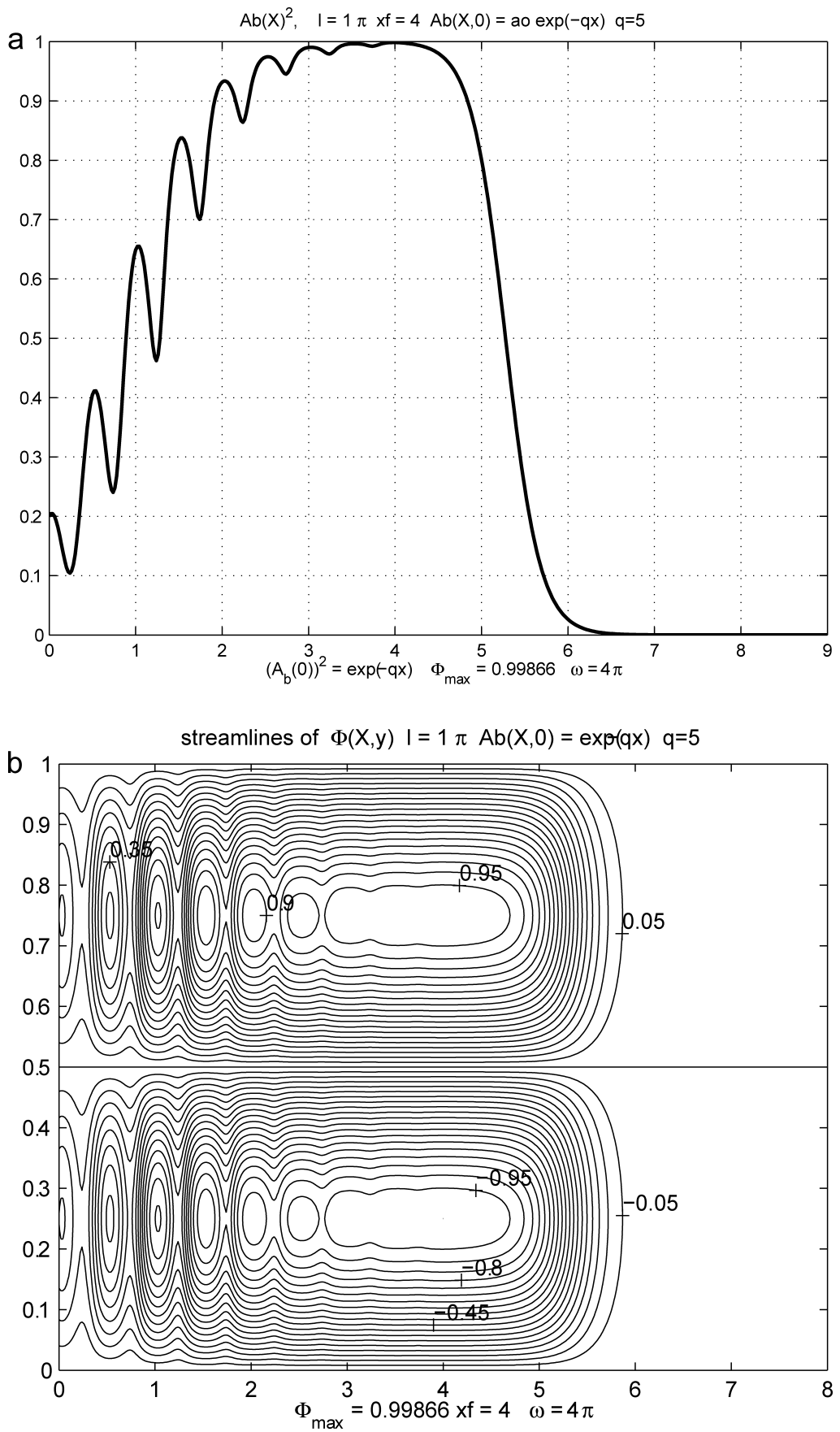

Figure 2. (a) The amplitude of the barotropic mode as a function of $X$. The front is at $X=4$ (in the scaled units described in the text). (b) The correction to the mean baroclinic streamfunction $\Phi_{T}$ which also yields the form of the large scale interface perturbation. 
of order $\Delta$ a range of wavenumbers of order $\Delta^{1 / 2}$ could be unstable. That suggests new "slow" space and time scales

$$
\tau=\left|\Delta^{1 / 2}\right| t, \quad \xi=\left|\Delta^{1 / 2}\right| x
$$

in addition to the slower space and time scales $T$ and $X$. A development similar to what we have already outlined leads to a new equation for the amplitude $A_{B}$,

$$
\begin{aligned}
& \left(\frac{\partial}{\partial T}+U_{B} \frac{\partial}{\partial X}\right) A_{B}-\sigma A_{B}+N A\left|A_{B}\right|^{2}=\mu \frac{\partial^{2} A_{B}}{\partial \xi^{2}}, \\
& \mu=\frac{2 r K^{2}}{\left(K^{2}+F\right)\left(2 F-K^{2}\right)}
\end{aligned}
$$

Note that $\mu$ is positive in the unstable region. There is necessary side condition required for the validity of (3.14a),

$$
\frac{\partial A_{B}}{\partial \tau}+U_{B} \frac{\partial A_{B}}{\partial \xi}=0
$$

The details of the derivation are straight forward but cumbersome and as a consequence of the discussion below, are largely irrelevant to our purpose as will be seen.

If we had a channel that was infinite in both positive and negative $x$ directions, i.e. in the absence of a fixed, physically significant origin, we could place ourselves in a frame moving with the speed $U_{B}$ and the advective term in (3.14a) would vanish. This would also be true if $U_{B}$ were zero. Note that neither of these cases is appropriate for the physical problem under consideration since we are interested in the downstream development from a particular, fixed origin and if $U_{B}$ were zero we would have no downstream development at all. However in that case, i.e. $U_{B}=0$, (3.14a) would be the so-called Ginzburg-Landau equation, independently derived and first introduced into the English language literature by Newell and Whitehead (1969) and Segel (1969). The behavior of solutions in the unbounded domain can be quite interesting but in our situation the constraint (3.14b) renders the equation problematic. If, say, $U_{B}$ is not zero, and this is the case we are concerned with, then (3.14b) implies that behind the front moving with $U_{B}$ the behavior of the amplitude as a function of $\tau$ and $\xi$ is completely determined by the behavior of the amplitude as a function of $\tau$ at $\xi=0$, i.e. the origin. As we have seen from the linear problem the natural time scale for the instability is on the time scale $T$ and if the amplitude is independent of $\tau$ at the origin it will remain so in the entire region behind the front and will also, therefore, be independent of $\xi$. Thus for our problem driven by weakly unstable fluctuations at the origin, i.e. $x=0$, the Ginzburg- Landau equation is irrelevant and our governing equation is just (3.9).

\section{The theory for $r=(\Delta)$}

We now consider the problem of downstream development when the friction is very small, i.e. when $r=\mathrm{O}(\Delta)$. In this limit the critical curve does not involve the shear and 
can most simply be written in terms of the parameter $F$, the square of the channel width to the deformation radius, namely,

$$
F=F_{C}=\frac{l^{2}+k^{2}}{2}
$$

which is parabolic and reaches a minimum as a function of $k$ at $k=0$. The theory to be developed will be a long wave theory for the instability. According to linear theory, in the absence of friction, the complex frequency satisfies,

$$
\omega-k U_{B}= \pm i\left(\frac{2 F-K^{2}}{2 F+K^{2}}\right)^{1 / 2} .
$$

Suppose that the $x$ wavenumber is small and we will expand it as

$$
k=\Delta^{1 / 2} k_{o}+\Delta k_{1}+\ldots
$$

while also,

$$
\begin{aligned}
& F=\frac{l^{2}}{2}+\Delta, \\
& \omega=\Delta^{1 / 2} \omega_{o}+\Delta \omega_{1}+\ldots
\end{aligned}
$$

For simplicity we will consider $\Delta$ always positive. Inserting these expansions in (4.2) yields,

$$
\begin{aligned}
& \omega_{o}=k_{o} U_{B}, \\
& \omega_{1}-k_{1} U_{B}=i \frac{k_{o}}{2^{1 / 2} l}\left(2-k_{o}^{2}\right)^{1 / 2} .
\end{aligned}
$$

Hence we can anticipate a development in which there can be an oscillation in space and time on the time scale $\Delta^{1 / 2}$ and a development on both the slower time and space scales $\Delta$. Note that according to linear theory, the maximum growth rate occurs for $k_{o}=1$. This suggests considering the streamfunction fields functions of

$$
\xi=\Delta^{1 / 2} x, \tau=\Delta^{1 / 2} t, X=\Delta x, T=\Delta t .
$$

With these new variables the governing equations (2.1) can be rewritten and expanded again, under the assumption verified a posteriori that the amplitude of the wave perturbation $\varepsilon$ is order $\Delta^{1 / 2}$.

At lowest order this yields for the wave amplitudes,

$$
\begin{aligned}
\varphi_{B}^{(0)} & =\frac{1}{2} A_{B} e^{i\left(k_{o} \xi-\omega_{o} \tau\right)} \sin l y+*, \\
\varphi_{T}^{(0)} & =0 . \\
k_{o} & =\frac{\omega_{o}}{U_{B}}
\end{aligned}
$$


So, we can think of the wavelength being determined by the frequency of the dominant fluctuation at the origin. Note that $A_{B}=A_{B}(X, T)$. At the next order, $\mathrm{O}\left(\Delta^{1 / 2}\right)$, we obtain,

$$
\begin{aligned}
\varphi_{B} & =\left(\frac{A_{B}(X, T)}{2} e^{i \theta}+*\right) \sin l y, \\
\varphi_{T} & =\Delta^{1 / 2}\left(\frac{4 i}{k_{o} U_{S}}\left[\frac{\partial}{\partial T}+U_{B} \frac{\partial}{\partial X}+\frac{r}{\Delta}\right] \frac{A_{B}}{2} e^{i \theta}+*\right) \sin l y+\Delta^{1 / 2} \Phi(y, X, T)
\end{aligned}
$$

At next order, $\mathrm{O}(\Delta)$, the equation governing the correction to the mean flow is determined,

$$
\begin{aligned}
\left(\frac{\partial}{\partial T}+U_{B} \frac{\partial}{\partial X}\right)\left(\frac{\partial^{2} \Phi}{\partial y^{2}}-2 F_{c} \Phi\right) & +\frac{r}{\Delta} \frac{\partial^{2} \Phi}{\partial y^{2}} \\
& =\frac{\varepsilon}{\Delta^{1 / 2}} \frac{2 F_{C}}{U_{S}} l \sin 2 l y\left[\frac{\partial}{\partial T}+U_{B} \frac{\partial}{\partial X}+\frac{2 r}{\Delta}\right]\left|A_{B}\right|^{2}
\end{aligned}
$$

while the same order determines the evolution equation for the wave amplitude. First, though, we note that as before we can search for solutions for the mean flow correction in the form,

$$
\Phi=P(X, T) \sin 2 l y
$$

so that our system of equations becomes the set of partial differential equations

$$
\begin{array}{r}
\left(\frac{\partial}{\partial T}+U_{B} \frac{\partial}{\partial X}\right) P+\frac{4}{5} \frac{r}{\Delta} P=-\frac{\varepsilon}{\Delta} \frac{2 F_{C}}{l U_{S}}\left(\frac{\partial}{\partial T}+U_{B} \frac{\partial}{\partial X}+2 \frac{r}{\Delta}\right)\left|A_{B}\right|^{2}, \\
\left(\frac{\partial}{\partial T}+U_{B} \frac{\partial}{\partial X}\right)^{2} A_{B}+\frac{3}{2} \frac{r}{\Delta}\left(\frac{\partial}{\partial T}+U_{B} \frac{\partial}{\partial X}\right) A_{B} \\
-\sigma^{2} A_{B}-\frac{2 \varepsilon}{\Delta^{1 / 2}} l^{2} k_{o}^{2} U_{s} A_{B} P=0, \\
\sigma^{2}=\frac{U_{s}^{2}}{2}\left\{l^{2}-k_{o}^{2}\right\} \frac{k_{o}^{2}}{l^{2}}-\frac{r^{2}}{2 \Delta^{2}}
\end{array}
$$

We can remove the parameters from $(4.9 \mathrm{a}, \mathrm{b})$ by the following rescalings,

$$
T^{\prime}=\sigma T, X^{\prime}=\sigma X / U_{B}, A_{B}=A_{o} A^{\prime}, P=P_{o} P^{\prime}, \gamma=\frac{r}{\Delta \sigma}
$$

where

$$
\varepsilon=\Delta^{1 / 2}, P_{o}=\frac{\sigma^{2}}{k_{o}^{2} U_{s} l}, A_{o}=\frac{\sigma}{k_{o} l}
$$


so, that after dropping primes on $T$ and $X$, our final amplitude equations become,

$$
\begin{aligned}
& \left(\frac{\partial}{\partial T}+\frac{\partial}{\partial X}\right)^{2} A_{B}+\frac{3}{2} \gamma\left(\frac{\partial}{\partial T}+\frac{\partial}{\partial X}\right) A_{B}-A-A P=0, \\
& \left(\frac{\partial}{\partial T}+\frac{\partial}{\partial X}\right) P+\frac{4}{5} \gamma P=-\left[\left(\frac{\partial}{\partial T}+\frac{\partial}{\partial X}\right)\left|A_{B}\right|^{2}+2 \gamma\left|A_{B}\right|^{2}\right]
\end{aligned}
$$

As was the case for the $r=\mathrm{O}(1)$ problem there is a single set of characteristics. Defining the characteristic coordinate $s$ by the differential relations

$$
\frac{d T}{d s}=1, \frac{d X}{d s}=1,
$$

$(4.11 \mathrm{a}, \mathrm{b})$ can be written as the set of first order ordinary differential equations

$$
\begin{aligned}
\frac{d A_{B}}{d s} & =-\gamma A_{B}+B, \\
\frac{d B}{d s} & =-\frac{\gamma}{2} B+\left(1+\gamma^{2} / 2\right) A_{B}-A_{B}\left|A_{B}\right|^{2}-R A_{B}, \\
\frac{d R}{d s} & =-\frac{4}{5} \gamma R+\frac{6}{5} \gamma\left|A_{B}\right|^{2}
\end{aligned}
$$

where

$$
P=-\left|A_{B}\right|^{2}-R
$$

This set of ordinary differential equations written along the characteristics, i.e. the same lines as shown in Figure 1, are of the form of the well known Lorenz equations. For a range of moderately small $\gamma$ the solutions have a chaotic behavior as a function of $s$. Figure 3 shows the behavior of the amplitude along four characteristics that are closely spaced at $s=0$. In the region behind the advancing front this corresponds to slightly different specified amplitudes at $X=0$, i.e. values with only slightly different starting values at the physical origin. The great sensitivity of chaotic systems like the one described by (4.13) means that as the distance along closely neighboring characteristics increases the chaotic divergence of the solution trajectories implies that the solutions will change by order one from one characteristic to its neighbor. Indeed, the solution of (4.13) can most easily and reliably be found by integrating those equations along each characteristic and, to find the solution for fixed time, it is only necessary to label the characteristic by its initial intersection with the $T$ axis as shown in Figure 4. In the region behind the front the solution to the characteristic equations, in our scaled units,

$$
\frac{d X}{d s}=1, \frac{d T}{d s}=1, \Rightarrow X=s, T=s+T_{o}
$$

where $T_{o}$ is the intersection of the characteristic with the $T$ axis at $X=0$. For a given $X$ and $T$, the solution is found for the value of the amplitude at fixed $T$ by evaluating the 


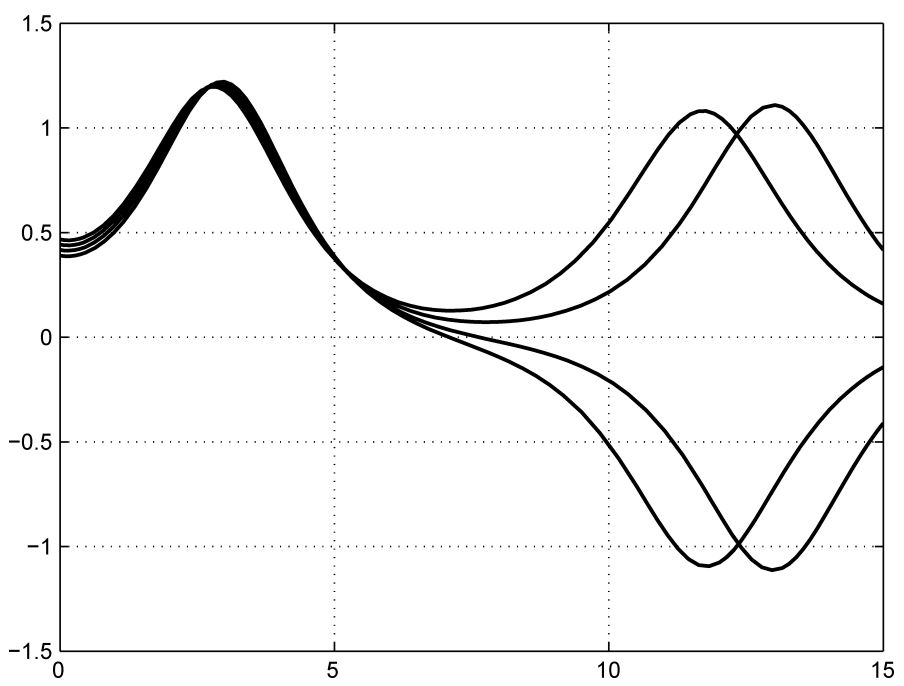

Figure 3. The solution for $A_{B}$ along four characteristics that, at $X=0$ are originally closely spaced. The chaotic nature of the dynamics along the characteristics shows that at moderately large values of $s$ the solutions diverge by an order one amount.

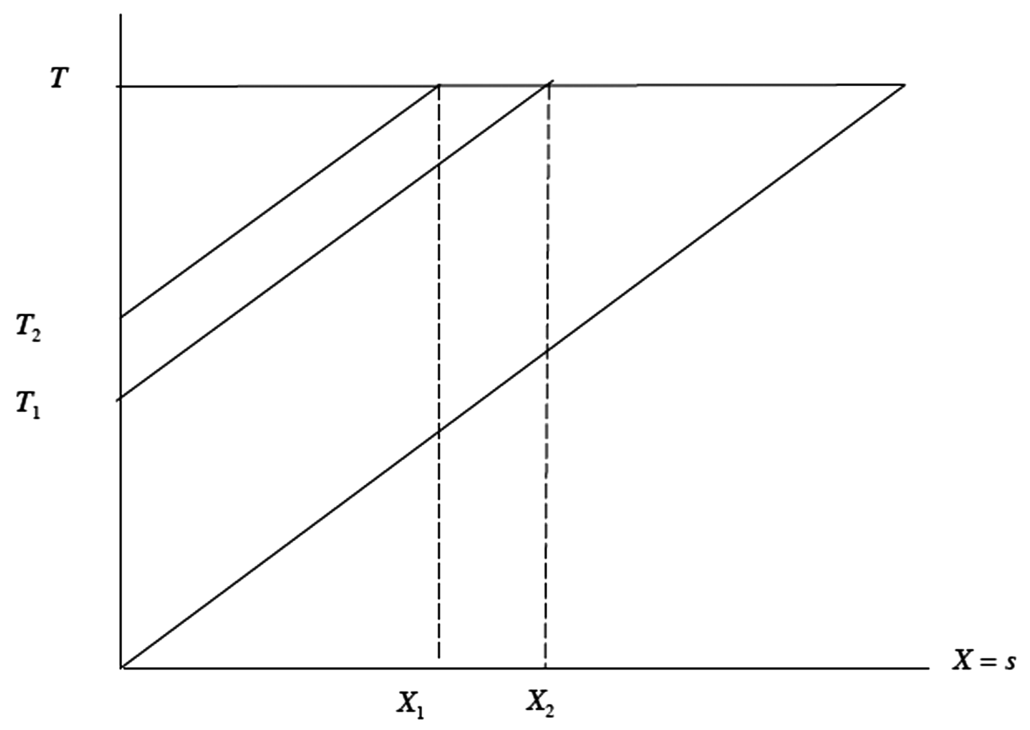

Figure 4. The solution at time $T$ is obtained from the solution along each of the characteristics intersecting the characteristics emanating from $s=X=0$. Even for small differences $T_{2}-T_{1}$ the variation along the line $T=$ constant can vary rapidly due to the chaotic behavior of the solution along the characteristics, e.g. the sensitivity to the initial conditions at the origin. 
amplitude on each characteristic intersecting the level line $T=$ constant whose starting value is simply $T_{o}=s-T$. This is easily done by integrating along each characteristic on a gridded line in $s$ (or $X$ ) and evaluating the intersection point, $s-T$. Even if the starting values of $T_{o}$ are close the chaotic character of the solutions along each characteristic will introduce very rapid changes of the solution at fixed $T$ from one characteristic to its neighbor and hence from one value of $X$ to the next. That is, the sensitivity of the solutions along the characteristics to very small changes in the starting values can lead to very large changes in the solution as a function of $X$ if the solution is carried far enough in $s$, i.e. in $X$, for the chaotic behavior to manifest itself. Figure 5 shows the response in $X$ of the wave amplitude at three times, $T=3,6$, and 10 for the region behind the front. In the example we have chosen there is no perturbation ahead of the front, i.e. at $T=0$ the perturbation is zero everywhere and the solution is forced by the condition at the origin,

$$
A_{B}(0, T)=1-e^{-T} .
$$

This forcing has been chosen since it is monotonic and so the oscillations seen in the solution are internally generated. For short times the solution is very smooth but at time goes on and the characteristics lengthen the chaotic behavior begins to manifest itself and rapid variations of the solution are clearly seen behind the front. At $T=10$ a second region of increasing gradient is emerging. For large time the forcing approaches a constant and the behavior along each characteristic will be nearly identical which is why the rapid behavior seems to be restricted to the region near the front. If instead of the condition (4.15) we impose the condition

$$
A_{B}(0, T)=a \sin \left(2 \pi T / T_{\text {period }}\right) .
$$

The solution for the forcing in (4.16) is shown in Figure 6 where the $T_{\text {period }}$ is 5 and $a=0.5$. The solution forcing is never monotonic and the manifestation of chaotic behavior is the appearance of rapid variations of amplitude which occur well behind the front demonstrating that it is not the front that excites the rapid variation but the sensitivity to slightly varying initial conditions from one characteristic to its neighbor.

These regions of rapid variations in amplitude with $X$ are thus regions that have relatively large meridional (i.e. $y$ ) velocities and rapid changes in those velocities. Normally in hyperbolic systems shocks occur when the characteristics of the problem overlap because of nonlinear bending of the characteristic curves. Instead, here the characteristics remain simple, indeed, they remain straight parallel lines. It is the chaotic character of the solutions along those characteristics and the divergence of the solutions on neighboring characteristics that provides the shrinking scales in the behavior of the solution. It is tempting to call such behavior "chaotic shocks" although they are not shocks in the traditional gas dynamics sense.

Of course, this rapid behavior is rapid on the "slow" scales. When the solution in $(X, T)$, becomes too rapid the dynamics on the original deformation radius scale of the carrier wave 

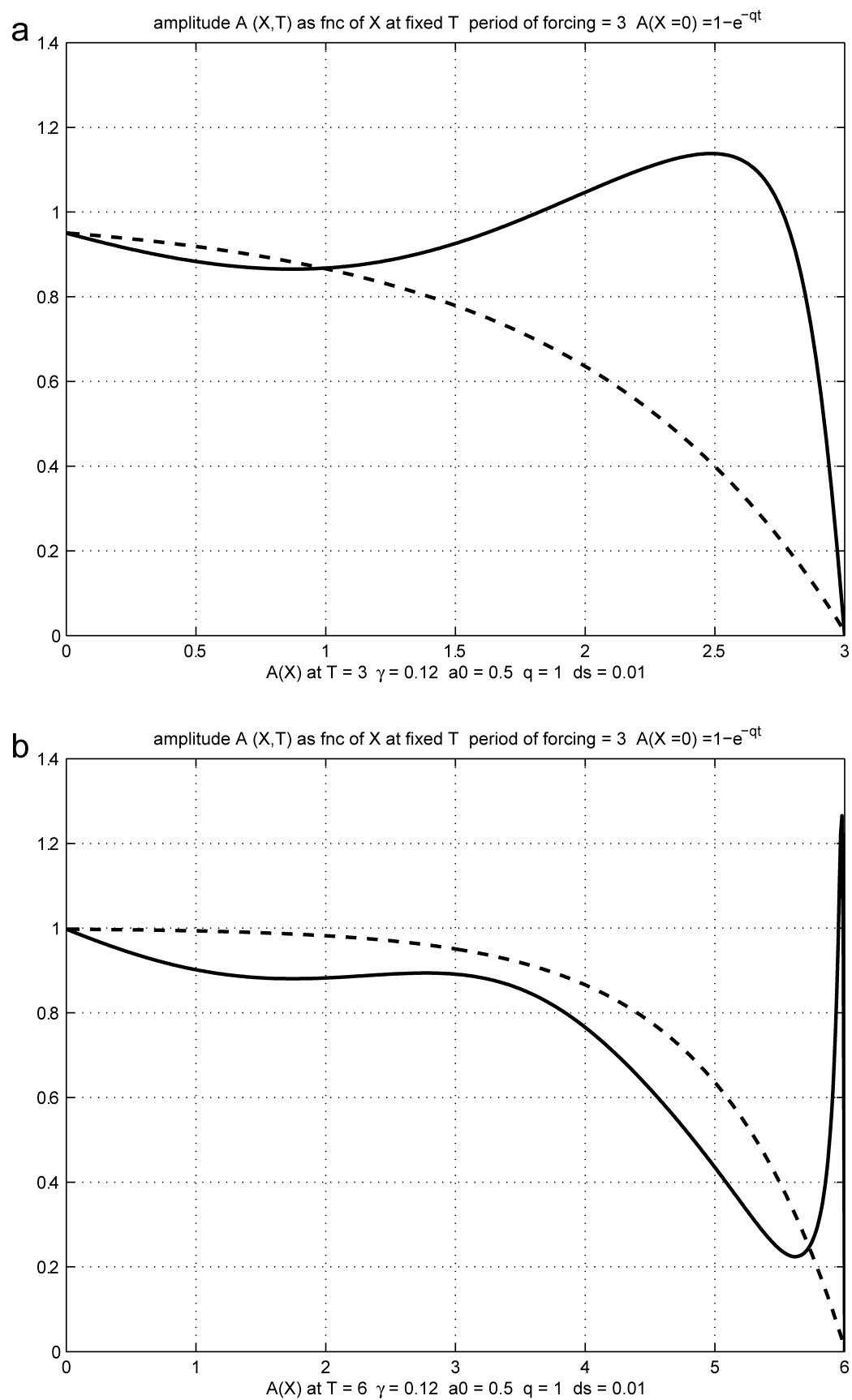

Figure 5. The amplitude behind the advancing front at three times (a) $T=3$, (b) $T=6$, (c) $T=10$. The rapid variation occurs when the length of the characteristic is large enough to manifest chaotic behavior. 


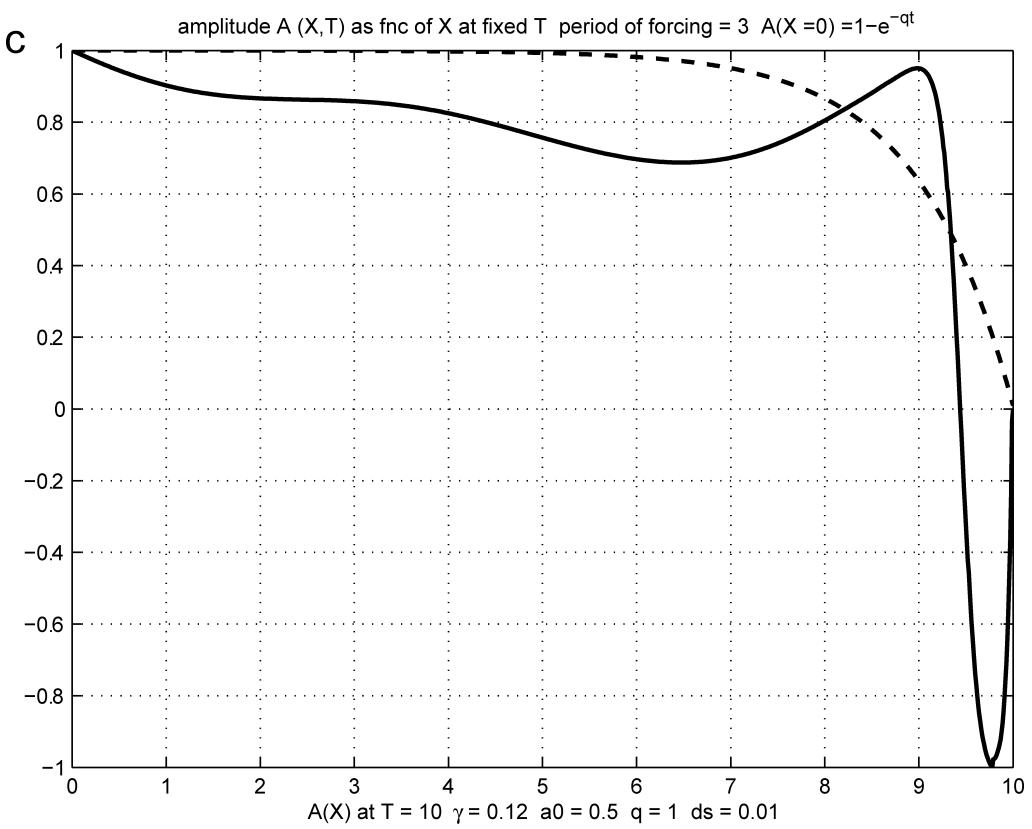

Figure 5. (continued)

will become intrinsically mixed with the long wave dynamics and one can speculate that this limits the scale collapse. It suggests a future study allowing the interaction of the two-scale dynamics in that limit.

\section{Conclusions and discussion}

Although heavily idealized, the model nevertheless exhibits a rich catalogue of behavior in its downstream development. The spatial development of the baroclinic instabilities in this simple model depends on the level of the intrinsic dissipation in the system. When the frictional time scale, i.e. the spin-down time, is of the same order as the advective time scale, so that the parameter $r$ is order one, the governing equation of long time and space scales is first order in both time and space. For slightly supercritical states the motion is also weakly nonlinear and analytic solutions are easily found by the method of characteristics. The spatial structure consists of a front propagating with the barotropic mean flow. The disturbance ahead of the front reflects the spatial distribution of the perturbation pre-existing at the initial instant. Behind the front the spatial structure reflects the temporal structure of the perturbation prescribed at the spatial origin of the flow until the amplitude reaches its finite amplitude equilibrium value. In the simple model presented here, in which the basic flow is uniform in the downstream direction, this equilibrium amplitude is independent of $x$ and the perturbation assumes the form of a zonal perturbation of the original basic flow. That 


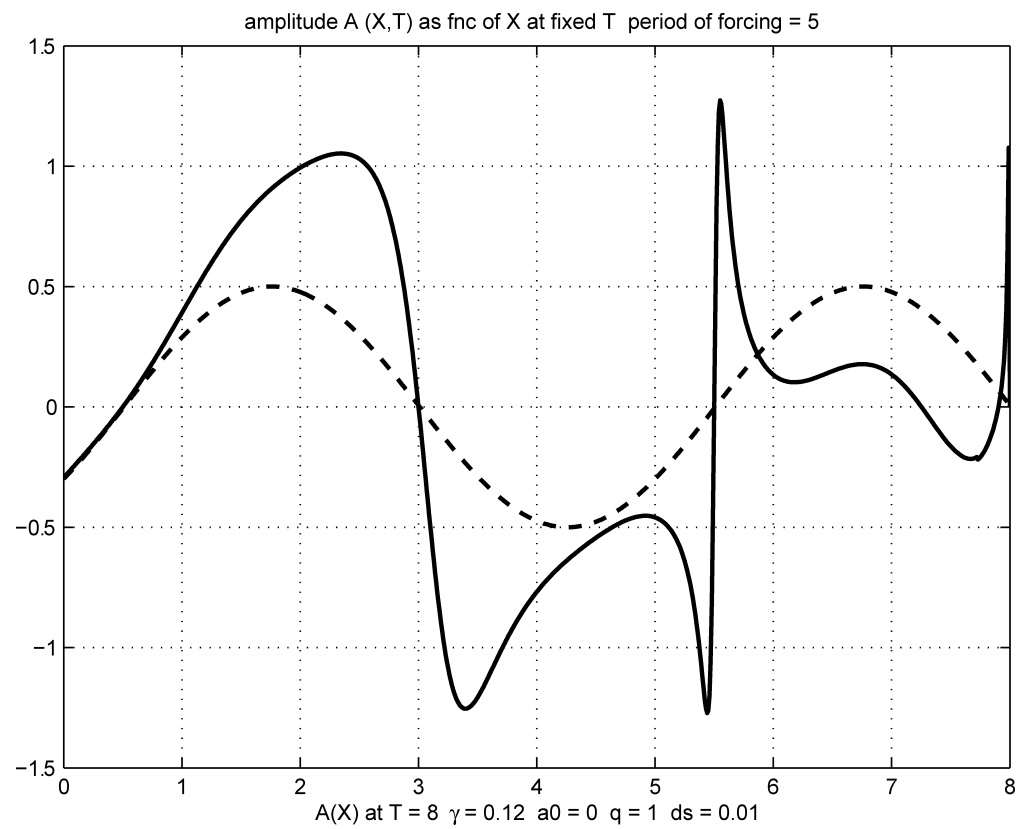

Figure 6. The solution for the amplitude as a function of $X$ when the amplitude at $X=0$ oscillates with $T$.

is evident in Figure 2b in the region between $X \sim 3.5$ and 4.5. In all cases the alteration of the interface has no net cross stream average so the reduction in the vertical shear that produces the equilibration does not imply a transfer of fluid between the layers, impossible in this adiabatic model.

When the frictional time scale is long, i.e. of the same order as the e-folding time of the slightly supercritical instability, the governing partial differential equation is second order although, again, the characteristics of the flow are determined by the barotropic advective velocity. The amplitude equations along each characteristic are essentially the equations of the Lorenz (1963) model and for a range of the friction parameter $\gamma$ show chaotic behavior along the characteristics. Since each characteristic has a slightly different initial condition if the amplitude at the spatial origin is a function of time, the solutions along the characteristics will diverge by an order one amount even if the starting conditions are nearly equal. This implies that smoothly varying conditions at the origin will give amplitude values at later fixed times, at some distance from the origin, that can change very rapidly from one characteristic to another, i.e. very rapidly in space. The rapidity is so great that it suggests a shock phenomenon even though the classical shock behavior of hyperbolic systems is impossible here since the characteristics are always straight parallel lines. The brusque change is due entirely to the chaotic behavior along the characteristics and chaotic shock seems an appropriate terminology for the phenomenon. 
The model described here is very simple and it is obvious that more complex physics needs to be added to be able to adequately describe the behavior of real oceanic systems, like the Gulf Stream or Kuroshio extension regions which have suggested this study. The presence of horizontal shear, non rigid lateral boundaries, more complex potential vorticity structure of the basic state, may all affect the ideas described here. It will be of interest to examine them in the future. Nevertheless, the transformation of chaotic behavior in the time domain to abrupt spatial change in the downstream direction would seem to be a general and robust property of hyperbolic systems with chaotic behavior along the characteristics applicable in many situations.

Acknowledgments. This research was supported in part by NSF Grant OCE 0925061. It is a special pleasure to be able to participate in this volume in honor of Melvin Stern and to acknowledge his generous and inspiring mentorship over the course of my own career. His interest in fluid mechanics and especially baroclinic instability helped inspire my own. I also thank Karl Helfrich for his help in aspects of the calculations of Section 4.

\section{REFERENCES}

Briggs, R. J. 1964. Electron Stream Interaction with Plasmas, MIT Press, Cambridge, Chapter 2, $8-46$.

Hocking, L. M., K. Stewartson and J. T. Stuart. 1972. A nonlinear burst in plane Poiseuille flow. J. Fluid Mech., 51, 705-736.

Lorenz, E. N. 1963. Deterministic nonperiodic flow. J. Atmos. Sci., 12, 130-141.

Newell, A. C. and J. A. Whitehead. 1969. Finite bandwidth, finite amplitude convection. J. Fluid Mech., 38, 279-304.

Pedlosky, J. 1976. Finite amplitude baroclinic disturbances in downstream varying currents. J. Phys. Oceanogr., 6, 335-344.

1987. Geophysical Fluid Dynamics, 2nd ed., Springer-Verlag, NY, 710 pp.

Pierrehumbert, R. T. and K. L. Swanson. 1995. Baroclinic instability. Ann. Rev. Fluid Mech., 27, 419-467.

Polvani, L. M. and J. Pedlosky. 1988. The effect of dissipation on spatially growing nonlinear baroclinic waves. J. Atmos. Sci., 45, 1977-1989.

Segel L. A. 1969. Distant side walls cause slow amplitude modulation of cellular convection. J. Fluid Mech., 38, 203-224.

Stewartson, K. and J. T. Stuart. 1971. A non-linear instability theory for a wave system in plane Poiseuille flow. J. Fluid Mech., 48, 529-546.

Waterman, S. N. and S. R. Jayne. 2011. Eddy-mean flow interactions in the along-stream development of a western boundary current jet: An idealized model study. J. Phys. Oceanogr., 41, 682-707.

Received: 29 November, 2010; revised: 9 May, 2011. 